\title{
Modelos potenciales de distribución geográfica y climática del complejo Amblyomma cajennense (Acari: Ixodidae), potencial vector de Rickettsia rickettsii en Colombia
}

\author{
Leidy Y. Acevedo-Gutiérrez¹, Luis E. Paternina², Andrés F. Londoño³, \\ Gabriel Parra-Henao ${ }^{4}$, Juan D. Rodas ${ }^{1}$
}

\footnotetext{
1 Grupo de Investigación en Ciencias Veterinarias Centauro, Universidad de Antioquia, Medellín, Colombia

2 Grupo de Investigaciones Biomédicas, Universidad de Sucre, Sincelejo, Colombia

3 Grupo de Investigación en Medicina Veterinaria (GIVET), Corporación Universitaria Lasallista, Medellín, Colombia

${ }^{4}$ Centro de Investigación en Salud para el Trópico (CIST), Universidad Cooperativa de Colombia, Santa Marta, Colombia
}

\begin{abstract}
Introducción. Las especies del complejo Amblyomma cajennense, potenciales vectores implicados en la transmisión de Rickettsia rickettsii en Centroamérica y Suramérica, presentan una amplia distribución geográfica en el neotrópico. En Colombia, todavía es incipiente el conocimiento sobre las especies de este complejo, su distribución y su ecología.

Objetivo. Explorar la ecología del complejo A. cajennense en Colombia a partir del estudio de su selección de hábitat y la generación de modelos potenciales de distribución geográfica y climática.

Materiales y métodos. Se utilizaron registros únicos de la presencia de estas garrapatas en el estudio de sus patrones de selección del hábitat, mediante el análisis factorial del nicho ecológico (Ecological Niche Factor Analysis, ENFA), y se construyeron modelos de distribución potencial con las metodologías MaxEnt y GARP.

Resultados. El complejo $A$. cajennense se encontró en las cinco regiones naturales de Colombia, con $64,71 \%$ de los registros procedentes de los departamentos de Antioquia, Cundinamarca y Huila. La selección del hábitat evidenció que estas garrapatas escogen preferentemente las áreas geográficas (ENFA: 96,03 \%) según sus condiciones de índice de estabilidad térmica y de precipitación en el trimestre más seco del año. Los modelos de distribución potencial indicaron la idoneidad del hábitat en 31 a $51 \%$ del área del país dependiendo del algoritmo empleado.

Conclusiones. En Colombia, el complejo A. cajennense presenta una distribución muy influenciada por el comportamiento en la selección del hábitat climático, característica que facilitó la creación de modelos de distribución. En futuros estudios de campo, se podrían validar los modelos presentados; se requieren, además, otros estudios genéticos para la identificación de especies del complejo y el manejo de sus potenciales zonas de hibridación en el país.
\end{abstract}

Palabras clave: ecosistema; ecología, garrapatas, vectores artrópodos, clima. doi: https://doi.org/10.7705/biomedica.v38i4.3885

Potential models of the geographic and climatic distribution of the Amblyomma cajennense complex (Acari: Ixodidae), a potential vector of Rickettsia rickettsii in Colombia

Introduction: The species of the Amblyoma cajennense complex, potential vectors involved in the transmission of Rickettsia rickettsii in Central and South America, have a wide geographical distribution in the Neotropics. In Colombia, the knowledge about these species, their distribution, and many aspects of their ecology is still limited.

Objective: To explore the ecology of $A$. cajennense complex in Colombia based on the study of its habitat selection, as well as the generation of potential geographic and climatic distribution models.

\footnotetext{
Contribución de los autores:

Leidy Y. Acevedo-Gutiérrez y Luis E. Paternina: recolección, análisis e interpretación de los datos y redacción del manuscrito Andrés F. Londoño: obtención de los datos primarios y revisión del documento

Gabriel Parra-Henao: capacitación en el análisis y revisión del documento

Juan D. Rodas: análisis de resultados, redacción y revisión del documento
} 
Materials and methods: We used unique records of these ticks to study the selection patterns of the climatic habitat using the Ecological Niche Factor Analysis (ENFA), and we built distribution models using the MaxEnt and GARP algorithms.

Results: The record of distributions showed that $A$. cajennense complex is present in all five natural regions of Colombia with $64.71 \%$ of the records found in the departments of Antioquia, Cundinamarca, and Huila. The analysis of their habitat selection showed that these ticks preferably select geographical areas (ENFA: $96.03 \%$ ) according to the isothermality conditions and the precipitation of the driest quarter of the year. The potential distribution models indicated habitat suitability in 31 to $51 \%$ of the area of the country depending on the algorithm.

Conclusions: The distribution of $A$. cajennense complex in Colombia is highly influenced by a climatebased habitat selection, a characteristic that favored the creation of distribution models. Field studies will allow the validation of the potential distribution models and further genetic studies will be required in the country to identify species within the complex and study their potential hybridization areas.

Keywords: Ecosystem; ecology; ticks, arthropod vectors, weather. doi: https://doi.org/10.7705/biomedica.v38i4.3885

En estudios recientes se ha demostrado que el taxón Amblyoma cajennense es un complejo de, al menos, seis especies de garrapatas: $A$. mixtum, $A$. patinoi, $A$. cajennense sensu stricto, $A$. interandinum, $A$. sculptum y $A$. tonelliae $(1,2)$. Esta reclasificación se basa en diferencias biológicas, reproductivas, morfológicas y genéticas, que distinguen a las diferentes especies del complejo $(1,2)$, el cual incluye garrapatas neotropicales de tres huéspedes, con una distribución geográfica que va desde el sur de Estados Unidos hasta el norte de Argentina, incluidas algunas islas del Caribe, y una gran variedad de hábitats (3). Esta garrapata infesta principalmente animales equinos y bovinos, aunque se ha reportado en otros muchos, entre ellos, el hombre (4).

Las garrapatas del complejo $A$. cajennense han sido vinculadas como vectores principales o potenciales de Rickettsia rickettsii (5), agente causal de la fiebre manchada de las Montañas Rocosas en Estados Unidos, la fiebre de Tobia en Colombia y la fiebre maculosa en Brasil, zoonosis considerada reemergente en el continente americano (6). Estas garrapatas también se han encontrado infectadas con otras bacterias del género Rickettsia, y con microorganismos como Theileria equi (7) y Coxiella burnetti (8), y se ha demostrado que pueden estar involucradas en el mantenimiento del ciclo del virus de la encefalitis equina venezolana (9). En Colombia, su presencia se ha registrado desde finales del siglo XIX, cuando Neumann se refirió a

Correspondencia:

Leidy Yoana Acevedo-Gutiérrez, Grupo de Investigación en Ciencias Veterinarias Centauro, Universidad de Antioquia, Calle 70 № 52-21, Medellín, Colombia

Teléfono: (574) 219 6593; fax:(574) 2196525

leidy.acevedo@udea.edu.co muestras procedentes del país depositadas en el Museo de París y en el de Hamburgo (1899), en tanto que, a principios de siglo, Lawrence Dunn la señaló como la garrapata más común en las zonas de Colombia que pudo visitar (10).

A pesar de las numerosas referencias de garrapatas del complejo $A$. cajennense en Colombia, muchos de estos estudios se refieren a la vigilancia parasitológica en animales de producción y, por ello, no se cuenta con información relevante sobre su biología en etapas inmaduras o sobre su dinámica poblacional (11). En el país, las garrapatas de este complejo tienen un gran impacto en veterinaria, pues son ectoparásitos de animales bovinos y equinos, en tanto que, en salud humana, su relevancia se evidenció a partir de 1941, cuando se las vinculó como vector potencial de $R$. rickettsii durante un brote e fiebre manchada en Tobia, departamento de Cundinamarca (12). Desde entonces, se han presentado brotes confirmados de la enfermedad en los departamentos de Cundinamarca, Antioquia y Córdoba (13-15), sin que se haya vinculado ninguna especie de garrapata como vector.

Los reportes previos al 2015 se refieren al complejo $A$. cajennense, dada la imposibilidad de reclasificar los registros disponibles en cualquiera de los taxones que componen el complejo. Solo a partir de ese año, comenzaron a presentarse reportes concretos sobre especies del complejo A. cajennense en Colombia: $A$. patinoi en Cundinamarca y $A$. mixtum en Arauca y Casanare $(16,17)$. El hallazgo reciente de $A$. patinoi, especie nombrada en honor al doctor Luis Patiño Camargo, con $R$. rickettsii en una localidad cercana al sitio del brote originalmente descrito por él, sugiere que esta garrapata es el potencial vector de esta bacteria en esa región del país (18). 
Dadas las grandes variaciones ecogeográficas y la posible presencia de diversas entidades del complejo A. cajennense en el territorio nacional, así como la escasa información actual sobre la distribución y el hábitat de este importante grupo de garrapatas en Colombia, se consideró de suma pertinencia explorar la ecología del complejo a partir del estudio de su selección de hábitat, así como generar modelos para dar cuenta de su potencial distribución geográfica y climática.

\section{Materiales y métodos}

\section{Área de estudio}

La región de estudio se limitó al área continental de Colombia, con una extensión de 1'141.748 $\mathrm{km}^{2}$, ubicada entre las coordenadas $4^{\circ} 13^{\prime} 30^{\prime \prime}$ S, $12^{\circ} 27^{\prime} 46^{\prime \prime} \mathrm{N}, 66^{\circ} 50^{\prime} 54^{\prime \prime}$ E y $79^{\circ} 0^{\prime} 23^{\prime \prime}$ O. Esta extensión, que abarca 10 provincias biogeográficas según la propuesta de regionalización del Neotrópico de Morrone, así como numerosas ecorregiones según lo establecido por el World Wildlife Fund $(18,19)$, se dividió en 1'333.948 pixeles con una resolución de 30 arcsec para el estudio del hábitat de las garrapatas del complejo A. cajennense.

\section{Registros de la presencia de las garrapatas}

Los registros de la presencia del complejo $A$. cajennense se obtuvieron de la literatura científica, utilizando como punto de inicio los reportes de 1980 y cubriendo hasta el 2015. Asimismo, se incluyeron registros provenientes de comunicaciones personales, revisiones de colecciones entomológicas y recolecciones hechas por los propios autores. El registro único se estableció con aquellos provenientes de una misma coordenada geográfica. Cuando los datos fueron obtenidos por los investigadores, las coordenadas de los sitios de recolección se obtuvieron por georreferenciación en campo utilizando el gacetero de poblaciones de Colombia y aquellos reportes publicados con suficiente información para llevar a cabo el procedimiento, y siguiendo las guías del Instituto de Investigación de Recursos Biológicos Alexander von Humboldt (20).

\section{Datos climáticos}

Los registros de la presencia de garrapatas del complejo $A$. cajennense se superpusieron sobre capas de información del índice diferencial normalizado de vegetación (Normalized Difference Vegetation Index, NDVI), de las 19 variables bioclimáticas y de las diferentes altitudes, con una resolución de 30 arcsec, obtenidas del portal
WorldClim $(21,22)$. Posteriormente, se recuperó la información ecológica asociada con cada registro de garrapatas, utilizando la herramienta Point Sampling Tool de QGIS 2.4 (QGIS Development Core Team), con el fin de obtener una matriz de datos ecológicos.

Una vez obtenida la matriz de datos, se seleccionó un grupo de variables mediante un análisis de componentes principales, según su grado de correlación, su carga con respecto a los componentes y su importancia ecológica a priori. Este grupo de variables se utilizó en el estudio del hábitat climático y en la generación de modelos de habitabilidad para este grupo de garrapatas en Colombia.

Las siguientes fueron las variables seleccionadas después de reducir las dimensiones en el análisis de componentes principales: la altitud (Alt), el índice de estabilidad térmica (Bio3), la temperatura máxima del mes más cálido (Bio5), la temperatura mínima del mes más frío (Bio6), la precipitación anual (Bio12) y la precipitación del trimestre más seco del año (Bio17). Todos los análisis estadísticos se hicieron con el lenguaje programático $\mathrm{R}$ ( $R$ Core Development Team).

\section{Selección del hábitat climático}

El análisis de selección de hábitat se hizo mediante el algoritmo ENFA implementado en el paquete adehabitatHS $(23,24)$, con el fin de calcular el aporte de las variables ambientales a la marginalidad y a la especialización. La marginalidad se define como el vector de diferencias estandarizadas entre la media del espacio ambiental de la región de interés y la media del espacio ambiental utilizado por la especie, y la especialización, como la razón entre la varianza del espacio ambiental disponible en la región y la varianza del espacio ambiental utilizado por la especie (25). La marginalidad se utiliza como un indicador de selección de hábitat y las especializaciones se utilizan como un referente de la estrechez de nicho o de preferencia de hábitat en un espacio ecológico multidimensional, en este caso, de seis dimensiones netamente abióticas.

\section{Modelos de distribución potencial del complejo Amblyomma cajennense}

En la creación de los modelos de distribución potencial de las garrapatas del complejo $A$. cajennense con base en las variables previamente 
seleccionadas, se emplearon dos algoritmos: el algoritmo de máxima entropía de MaxEnt v3.3.3k (26) y el algoritmo genético de GARP v1.1.6 (27). El algoritmo MaxEnt se ejecutó con un submuestreo de bootstrap, 100 réplicas para la creación y una para la validación ( $20 \%$ de los puntos), sin extrapolación ni clamping (28), y el modelo binario final se obtuvo utilizando el umbral de probabilidad mínima en los datos de calibración. Por otro lado, en el algoritmo GARP se hicieron 100 réplicas, 1.000 iteraciones, una validación con el $20 \%$ de los puntos, una medida de omisión extrínseca y un umbral de omisión alto. Los diez mejores modelos (best subset) de GARP se sumaron para obtener el modelo binario final.

Los modelos se calibraron para los polígonos creados con los puntos de presencia a una distancia de $100 \mathrm{~km}$ y se proyectaron para toda el área de Colombia. Para la validación de los modelos, se calculó la curva operativa del receptor (Receiver Operating Characteristic, ROC) parcial (29) en el programa Tool for Partial-ROC (Narayani Barve 2008, Biodiversity Institute, Lawrence, KS).

\section{Resultados}

\section{Distribución del complejo Amblyomma cajennense en Colombia}

Se obtuvieron 74 registros de presencia compilados a partir de un total de 101 reportes del complejo $A$. cajennense y distribuidos en 13 departamentos del país: Antioquia, Arauca, Caldas, Casanare, Cesar, Córdoba, Cundinamarca, Huila, Magdalena, Meta, Sucre, Tolima y Valle del Cauca (cuadro suplementario 1).

Según la distribución geográfica de los registros de presencia, Antioquia presentó la mayor cantidad de registros únicos, seguida por Cundinamarca y Huila, que, en su conjunto, representaron el 64,7 $\%$ de todos los registros compilados. Por otro lado, el $74,1 \%$ de los registros de presencia se concentró en las áreas de bosque seco del valle del Magdalena, bosques húmedos de Magdalena y Urabá, y bosque montano del valle del Magdalena, en zonas con una cobertura de bosque de hoja ancha, sabanas, y mosaicos de cultivos y vegetación natural (cuadro 1).

\section{Análisis de selección del hábitat climático del complejo Amblyomma cajennense}

En general, el espacio climático ocupado por las garrapatas del complejo $A$. cajennense parece definirse por zonas donde no se presentan cambios bruscos de temperatura (alto índice de estabilidad térmica), relativamente secas (baja precipitación), altas temperaturas máximas durante los periodos cálidos (media: $31,17^{\circ} \mathrm{C}$ ), elevadas temperaturas mínimas durante el mes más frío del año (media: $20,19^{\circ} \mathrm{C}$ ) y, preferiblemente, ubicadas en tierras bajas (media: 472,9 m), aunque se han registrado poblaciones de estas garrapatas hasta los 1.771 metros de altitud (cuadro 2).

EI ENFA evidenció que las garrapatas de este complejo de especies ocupan el mismo tipo de espacio climático disponible en Colombia, independientemente de la especie del complejo a la que puedan pertenecer. El ENFA permitió condensar el $96,03 \%$ de los datos en tres ejes (eje 1: $79,9 \%$, eje 2: $8,87 \%$ y eje 3: 7,26 $\%)$, lo que evidencia una gran relación entre la distribución geográfica y la distribución climática de los registros. El biplot de los dos primeros factores del ENFA (figura 1) ilustra claramente el hábitat ocupado por las garrapatas del complejo A. cajennense (área gris oscura) en relación con el hábitat climático disponible en Colombia (área gris clara), destacándose la variable del índice de estabilidad térmica como la más importante para la selección del hábitat (marginalidad: 0,57), seguida por la precipitación del trimestre más seco (especialización 1: -0,82) y la de temperatura mínima del mes más frío (especialización 2: -0,81), todas ellas sujetas a preferencias climáticas (figura 1, cuadro 2).

La ilustración del hábitat bidimensional de las garrapatas empleando las variables más importantes según el ENFA (precipitación del trimestre más seco e índice de estabilidad térmica), permitió confirmar la importancia de ambas variables y evidenció que entre 72 y $93 \%$ de las observaciones se encontraba por encima del $80 \%$ del índice de estabilidad térmica y entre los 19 y los $314 \mathrm{~mm}$ de precipitación durante el trimestre más seco del año, con muy escasas observaciones remotas $(3 / 74 ; 4,16 \%)$ (figura 1$)$.

\section{Modelos de distribución geográfica potencial}

Los modelos binarios de MaxEnt (umbral de probabilidad mínima en datos de calibración: $5,8 \%$ ) y de GARP, revelaron la idoneidad del hábitat para este complejo de especies en cuatro regiones naturales de Colombia, aunque la mayor parte de las áreas climáticamente adecuadas se encuentra principalmente en las regiones Caribe, Andina y de la Orinoquia, y en 
Cuadro 1. Registros georreferenciados únicos del complejo Amblyomma cajennense en Colombia según la división política y las ecorregiones definidas por el World Wildlife Fund (WWF)

\begin{tabular}{|c|c|c|c|}
\hline Departamento & Registros & Ecorregión & Registros \\
\hline \multirow[t]{3}{*}{ Antioquia } & 15 & Bosque montano del valle del Magdalena & 2 \\
\hline & & Bosque húmedo del Magdalena-Urabá & 9 \\
\hline & & Bosque montano andino noroccidental & 4 \\
\hline Arauca & 1 & Bosque seco del Apure-Villavicencio & 1 \\
\hline Caldas & 1 & Bosque húmedo del Magdalena-Urabá & 1 \\
\hline Casanare & 1 & Llanos & 1 \\
\hline Cesar & 1 & Bosque montano de Santa Marta & 1 \\
\hline Córdoba & 8 & Bosque húmedo del Magdalena-Urabá & 8 \\
\hline \multirow[t]{2}{*}{ Cundinamarca } & 15 & Bosque seco del valle del Magdalena & 11 \\
\hline & & Bosque montano del valle del Magdalena & 4 \\
\hline \multirow[t]{2}{*}{ Huila } & 12 & Bosque seco del valle del Magdalena & 9 \\
\hline & & Bosque montano del valle del Magdalena & 3 \\
\hline Magdalena & 1 & Matorrales xéricos de Guajira-Barranquilla & 1 \\
\hline \multirow[t]{2}{*}{ Meta } & 6 & Bosque seco del Apure-Villavicencio & 3 \\
\hline & & Llanos & 3 \\
\hline Sucre & 3 & Matorrales xéricos de Guajira-Barranquilla & 3 \\
\hline \multirow[t]{2}{*}{ Tolima } & 9 & Bosque seco del valle del Magdalena & 8 \\
\hline & & Bosque montano del valle del Magdalena & 1 \\
\hline Valle del Cauca & 1 & Bosque seco del valle del Cauca & 1 \\
\hline Total & 74 & & \\
\hline
\end{tabular}

Cuadro 2. Descripción general del hábitat climático del complejo Amblyomma cajennense en Colombia

\begin{tabular}{|c|c|c|c|c|c|c|}
\hline Variable & Media & Mínimo & Máximo & Mar & Spe1 & Spe2 \\
\hline Índice de estabilidad térmica (\%) (iso) & 86,37 & 72,0 & 93,0 & 0,57 & 0,16 & 0,52 \\
\hline $\begin{array}{l}\text { Temperatura }\left({ }^{\circ} \mathrm{C}\right) \text { máxima del mes más cálido } \\
(\text { maxtwm })\end{array}$ & 31,17 & 23,2 & 35,4 & 0,52 & $-0,40$ & 0,12 \\
\hline $\begin{array}{l}\text { Temperatura }\left({ }^{\circ} \mathrm{C}\right) \text { mínima del mes más frío } \\
(\text { mintcm) }\end{array}$ & 20,19 & 13,2 & 23,0 & 0,54 & 0,25 & $-0,81$ \\
\hline Precipitación anual (mm) (prean) & 1814,89 & 790 & 3942 & 0,25 & 0,15 & 0,20 \\
\hline $\begin{array}{l}\text { Precipitación del trimestre más seco (predriq) } \\
(\mathrm{mm})\end{array}$ & 168,40 & 19 & 314 & 0,12 & $-0,82$ & 0,02 \\
\hline Altitud (alt) (msnm) & 472,93 & 8 & 1771 & 0,2 & 0,21 & 0,17 \\
\hline
\end{tabular}

menor grado, en la región Pacífica y en la de la Amazonia. En los dos modelos se mantuvieron las predicciones de idoneidad climática en los valles interandinos, los llanos orientales y las sabanas de la región Caribe como sitios con una gran probabilidad de habitabilidad, en tanto que quedaron excluidos o tuvieron poca probabilidad de idoneidad climática los departamentos de Amazonas, Boyacá, Guainía, Putumayo y Vaupés (figura 2). El modelo GARP fue más conservador y ajustado a la distribución de los registros con predicciones de idoneidad de hábitat en el $31 \%$ del área continental de Colombia, en tanto que el modelo de MaxEnt predijo una idoneidad del hábitat en el $51 \%$ del territorio nacional (figura 2). En la evaluación de los dos modelos, se obtuvo una ROC parcial promedio de $1,9 \quad(p<0,001)$ y una sensibilidad general de $100 \%$ (74/74 puntos totales).

\section{Discusión}

El complejo A. cajennense está compuesto por seis entidades biológica, genética y morfológicamente diferenciadas $(1,2,30,31)$. Recientemente, Estrada- 

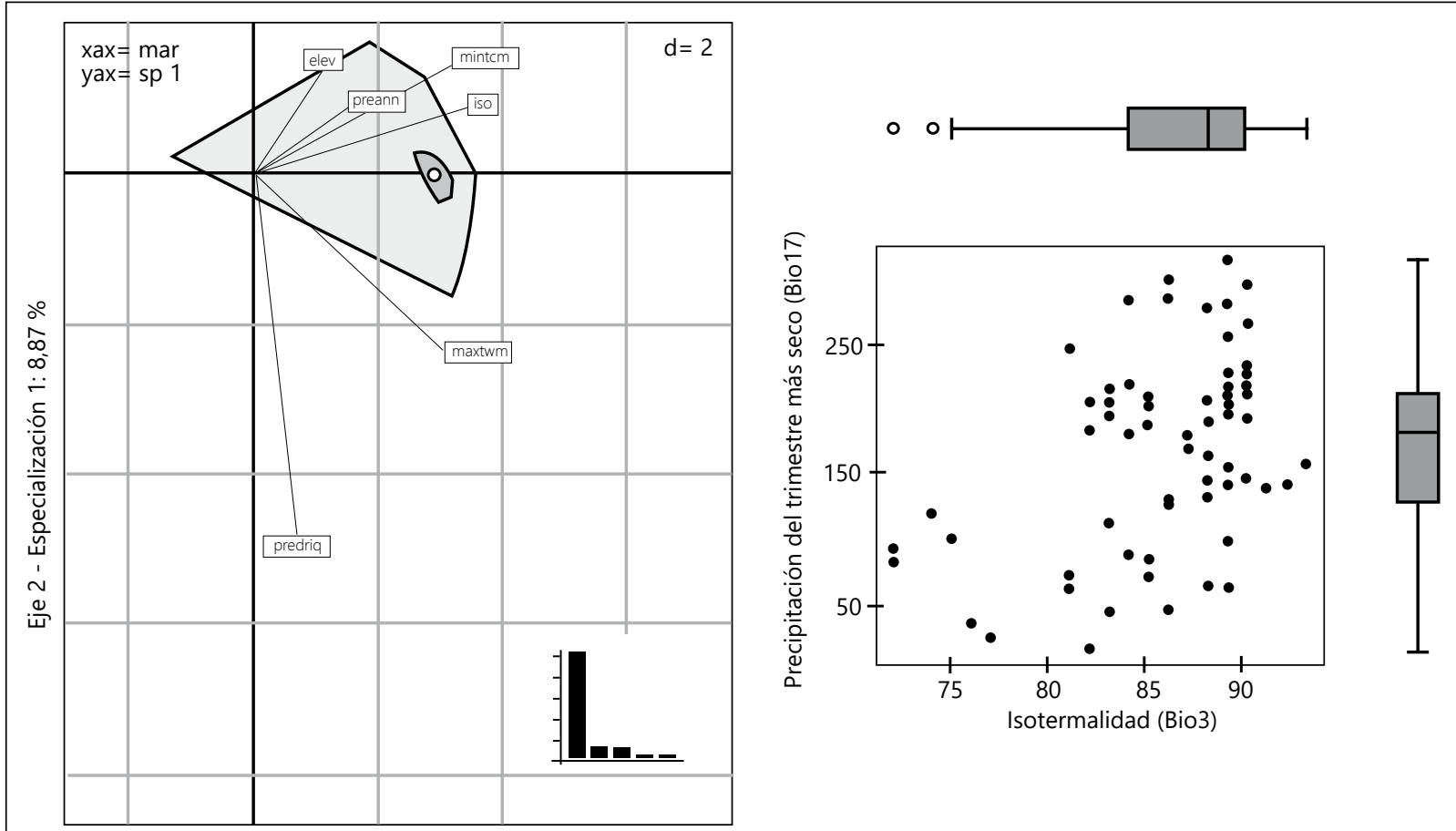

Eje 1 - Marginalidad: $79,9 \%$

Figura 1. Resultados del análisis factorial del nicho ecológico. El biplot (izquierda) ilustra el hábitat climático de estas garrapatas (gris oscuro) en relación con el espacio ecológico disponible (gris claro), en tanto que el hábitat bidimensional (derecha) muestra la distribución de los registros en relación con las variables más importantes según el ENFA.

Mar: marginalidad; Sp: especialización; elev: elevación; preann: precipitación anual; iso: índice de estabilidad térmica; mintcm: temperatura mínima del mes más frío; maxtwm: temperatura máxima del mes más cálido; predriq: precipitación del trimestre más seco
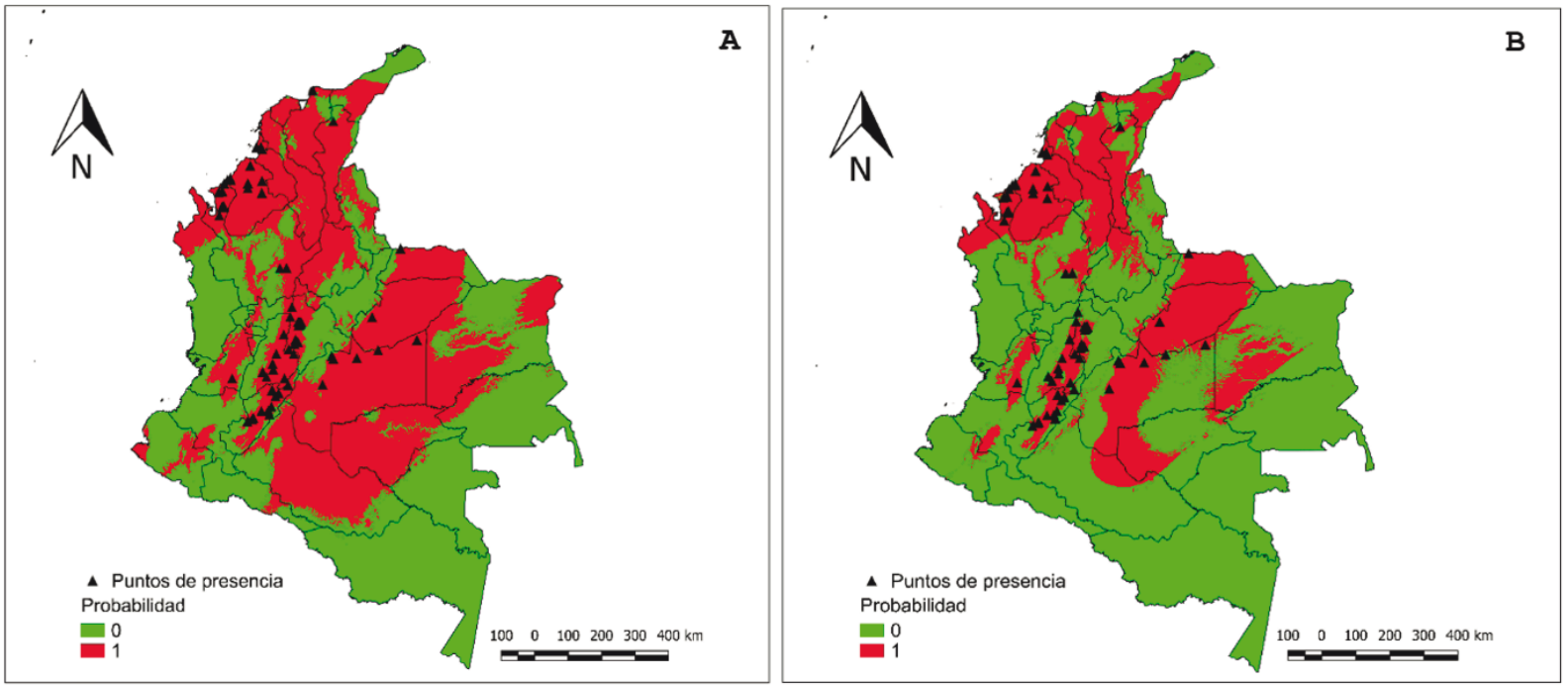

Figura 2. Modelos binarios de distribución potencial del complejo Amblyomma cajennense según los algoritmos MaxEnt (A) y GARP (B). Las áreas en rojo (1) indican idoneidad del hábitat climático para estas garrapatas, en tanto que las áreas en verde (0) denotan zonas climáticas no habitables 
Peña, et al., determinaron diferencias en cuanto a la ocupación del hábitat en función de la temperatura de superficie del suelo y del índice diferencial de vegetación normalizado, para cuatro especies de este complejo: $A$. mixtum y $A$. cajennense s.s., que presentan una distribución alopátrica, y $A$. sculptum y $A$. tonelliae, que presentan una distribución parapátrica. Estos autores, además, generaron modelos de habitabilidad que evidenciaron que el país presenta áreas climáticamente adecuadas para dos de las especies del complejo analizadas: A. cajennense s.s. en la Amazonia, la Orinoquia y los valles interandinos, y $A$. mixtum, la cual presenta probabilidades de habitabilidad en las costas del Pacífico y en la región Caribe (32).

El ENFA evidenció que el complejo $A$. cajennense en Colombia exhibe predilecciones climáticas comunes que permiten establecer las variables del índice de estabilidad térmica (Bio3) y precipitación del periodo más seco del año (Bio17) como los dos mejores descriptores del espacio ocupado por estas garrapatas. Según estos resultados, las garrapatas del complejo seleccionan espacios geográficos con gran estabilidad climática (índice de estabilidad térmica>72 \%) y prefieren zonas con pocas precipitaciones durante el periodo más seco del año (pretrims $<314 \mathrm{~mm}$ ), espacio climático bidimensional que permite representar adecuadamente la distribución del 95,9 \% de los registros de Colombia (71/74 puntos).

Si bien es cierto que algunas especies del complejo presentan las preferencias climáticas mencionadas en los análisis de Estrada-Peña, et al., la evidencia genética apunta a que la especiación del complejo A. cajennense respondió a eventos vicariantes recientes que interrumpieron áreas ya ocupadas por poblaciones ancestrales de estas garrapatas $(1,32)$. Ello explicaría por qué las poblaciones actuales de este complejo en Colombia han retenido los patrones ancestrales de selección del hábitat, tal como indican los resultados del ENFA en esta región del norte de Suramérica. Según la hipótesis de especiación propuesta por Beati, et al., el ascenso de los Andes en el norte de Suramérica pudo haber ocasionado la separación de las especies $A$. mixtum, $A$. cajennense s.s. y $A$. patinoi hace 3 a 6 millones de años y, dado que los Andes pueden considerarse una barrera semipermeable en zonas de moderada y baja elevación, es probable que existan zonas de simpatría para especies del complejo donde, incluso, puedan presentarse fenómenos de hibridación (1).
En cuanto a los modelos obtenidos de distribución potencial, estos indican que, al menos, un tercio del área continental del territorio nacional puede considerarse idóneo para el complejo $A$. cajennense. De hecho, los reportes de $A$. patinoi en Cundinamarca y $A$. mixtum en Arauca y Casanare(16), además de capturas recientes de garrapatas de este complejo en municipios que ofrecen idoneidad climática para ellas, tales como Neira en Caldas, Nunchía y Yopal en Casanare, y San Juan de Arama en Meta (33), permiten confirmar la utilidad de los modelos de distribución potencial aquí presentados. Estos coinciden con otros estudios realizados en Costa Rica, Brasil y Argentina, donde se han hecho estudios a partir de la ecología del complejo o de sus especies, en el sentido de que algunas características, como los climas cálidos o semiáridos y sin humedad extrema, favorecen la presencia de especies del complejo $(32,34,35)$.

Es de gran importancia resaltar que los modelos presentados reflejan proyecciones geográficas de variables abióticas; esto significa que la habitabilidad de un área determinada puede verse afectada por variables bióticas o abióticas no incluidas en nuestros análisis, que pueden modificar dicha habitabilidad. Por ejemplo, la humedad relativa (variable abiótica no analizada) puede afectar la supervivencia de poblaciones de estas garrapatas $(36,37)$, razón por la cual es probable que estas no hayan sido avistadas frecuentemente en zonas muy húmedas del Pacífico y la Amazonia colombiana.

Los patrones comunes de selección de hábitat del complejo $A$. cajennense y la semipermeabilidad de los Andes colombianos en algunos puntos geográficos, podrían explicar el hallazgo de $A$. mixtum (de distribución básicamente transandina) en Arauca y Casanare (16), localidades cisandinas que se encuentran muy por fuera del rango de distribución geográfica aceptada para esta especie $(1,2)$, aunque aparezcan como habitables por garrapatas de este complejo según los modelos de distribución potencial aquí presentados. Los hallazgos del presente estudio podrían considerarse complementarios a los presentados por Estrada-Peña, et al. en el 2014, ya que estos se restringieron a los patrones de selección y distribución del complejo $A$. cajennense en una escala geográfica más local, donde la distribución de los taxones no es necesariamente alopátrica o parapátrica (32). 
Los resultados del presente estudio parecen indicar que, indistintamente de la especie, las garrapatas del complejo $A$. cajennense en Colombia podrían compartir parte del espacio climático, facilitando la creación de modelos de distribución potencial cuya utilidad debe ser evaluada en estudios de campo.

Por último, se requieren estudios de tipificación molecular de las garrapatas del complejo para establecer los límites geográficos de las especies presentes en Colombia, evaluar las relaciones entre sus poblaciones y determinar el papel de cada una de ella en los ciclos de transmisión local de $R$. rickettsiia en poblaciones humanas.

\section{Agradecimientos}

A Colciencias, por el apoyo a través del proyecto con código 111549326228 del 2011, y al programa de sostenibilidad 2013-2014 de la Universidad de Antioquia. A Colciencias, por el apoyo a LEP (convocatoria 528/2011) y AFLB (convocatoria $511 / 2010)$ por medio de becas de doctorado nacionales, y al programa Jóvenes Investigadores, por el apoyo a LYAG. A Marylin Hidalgo, por los registros de Cundinamarca, a Eduar Elías Bejarano de la Universidad de Sucre, por el suministro de especímenes de Magdalena, y a Agustín Góngora de la Universidad de los Llanos por el suministro de garrapatas de la Orinoquia.

\section{Conflicto de intereses}

Los autores declaran no tener conflicto de intereses.

Financiación

Colciencias, proyecto 111549326228.

\section{Referencias}

1. Beati L, Nava S, Burkman EJ, Barros-Battesti DM, Labruna MB, Guglielmone AA, et al. Amblyomma cajennense (Fabricius, 1787) (Acari: Ixodidae), the Cayenne tick: Phylogeography and evidence for allopatric speciation. BMC Evol Biol. 2013;13:267. https://doi.org/10.1186/14712148-13-267

2. Nava S, Beati L, Labruna MB, Cáceres AG, Mangold AJ, Guglielmone AA. Reassessment of the taxonomic status of Amblyomma cajennense (Fabricius, 1787) with the description of three new species, Amblyomma tonelliae n. sp., Amblyomma interandinum n. sp. and Amblyomma patinoin. sp., and reinstatement of Amblyomma mixtum, and Amblyomma sculptum. Ticks Tick Borne Dis. 2014;5:252276. https://doi.org/10.1016/j.ttbdis.2013.11.004

3. Estrada-Peña A, Guglielmone AA, Mangold AJ. The distribution and ecological 'preferences' of the tick Amblyomma cajennense (Acari: Ixodidae), an ectoparasite of humans and other mammals in the Americas. Ann Trop Med Parasitol. 2004;98:283-92. https://doi. org/10.1179/000349804225003316
4. Nava S, Guglielmone AA. A meta-analysis of host specificity in Neotropical hard ticks (Acari: Ixodidae). Bull Entomol Res.2013;103:216-24. https://doi.org/10.1017/S00 0748531 2000557

5. Labruna MB, Mattar S, Nava S, Venzal JM, Dolz G, Abarca K, et al. Rickettsioses in Latin America, Caribbean, Spain and Portugal. Rev MVZ Córdoba. 2011;16:2435-57.

6. Parola P, Labruna MB, Raoult D. Tick-borne rickettsioses in America: Unanswered questions and emerging diseases. Curr Infect Dis Rep. 2009;11:40-50. https://doi.org/10.1007/ s11908-009-0007-5

7. Scoles GA, Ueti MW. Amblyomma cajennense is an intrastadial biological vector of Theileria equi. Parasit Vectors. 2013;6:306. https://doi.org/10.1186/1756-3305-6306

8. Sanders DM, Parker JE, Walker WW, Buchholz MW, Blount K, Kiel JL. Field collection and genetic classification of tick-borne Rickettsiae and Rickettsiae-like pathogens from South Texas: Coxiella burnetii isolated from fieldcollected Amblyomma cajennense. Ann N Y Acad Sci. 2008;1149:208-11. https://doi.org/10.1196/annals.1428.022

9. Linthicum KJ, Logan TM, Bailey CL, Gordon SW, Peters CJ, Monath TP, et al. Venezuelan Equine Encephalomyelitis Virus infection in and transmission by the tick Amblyomma cajennense (Arachnida: Ixodidae). J Med Entomol. 1991;28: 405-9. https://doi.org/10.1093/jmedent/28.3.405

10. Dunn H. Notes on some insects and other arthropods affecting man and animals in Colombia. Am J Trop Med Hyg. 1929;1:493-508. https://doi.org/10.4269/ajtmh.1929.s1-9.493

11. López G, Zúñiga I, Villar C, Osorio D. Distribución de garrapatas en 25 municipios de departamento de Antioquia. Rev ICA. 1985;20:40-4.

12. Patino L. Nuevas observaciones sobre un tercer foco de fiebre petequial (maculosa) en el hemisferio americano. Boletín Oficina Sanitaria Panamericana. 1941;20:1112-24.

13. Hidalgo M, Orejuela L, Fuya $P$, Carrillo $P$, Hernández J, Parra E, et al. Rocky mountain spotted fever, Colombia. Emerg Infect Dis. 2007;13:1058-60. https://doi.org/10.3201/ eid1307.060537

14. Acosta J, Urquijo L, Díaz A, Sepúlveda M, Mantilla G, Heredia D, et al. Brote de Rickettsia Rickettsii en Necoclí, Antioquia, Colombia, 2006. Inf Quinc Epidemiol Nac. 2006; $11: 161-76$

15. Hidalgo M, Miranda J, Heredia D, Zambrano P, Vesga JF, Lizarazo D, et al. Outbreak of Rocky Mountain spotted fever in Córdoba, Colombia. Mem Inst Oswaldo Cruz. 2011;106:117-8. https://doi.org/10.1590/S0074-027620110 00100019

16. Rivera-Páez FA, Labruna MB, Martins TF, Sampieri BR, Camargo-Mathias MI. Amblyomma mixtum Koch, 1844 (Acari: Ixodidae): First record confirmation in Colombia using morphological and molecular analyses. Ticks Tick Borne Dis. 2016;7:842-8. https://doi.org/10.1016/j.ttbdis.2016. 03.020

17. Faccini-Martínez AA, Costa FB, Hayama-Ueno TE, Ramírez-Hernández A, Cortés-Vecino JA, Labruna MB, et al. Rickettsia rickettsii in Amblyomma patinoi ticks, Colombia. Emerg Infect Dis. 2015;21:537-9. https://doi. org/10.3201/eid2013.140721 
18. Morrone JJ. Biogeographic areas and transition zones of Latin America and the Caribbean islands based on panbiogeographic and cladistic analyses of the entomofauna. Annu Rev Entomol. 2006;51:467-94. https:// doi.org/10.1146/annurev.ento.50.071803.130447

19. Olson DM, Dinerstein E, Wikramanayake ED, Burgess ND, Powell GV, Underwood EC, et al. Terrestrial ecoregions of the world: A new map of life on earth a new global map of terrestrial ecoregions provides an innovative tool for conserving biodiversity. BioScience. 2001;51:933-8. https:// doi.org/10.1641/0006-3568(2001)051[0933:TEOTWA]2.0. $\mathrm{CO} ; 2$

20. Sua S, Mateus RD, Vargas JC. Georreferenciación de registros biológicos y gacetero digital de localidades. Bogotá D.C.: Instituto de Investigación de Recursos Biológicos Alexander von Humboldt.; 2004. p. 69.

21. Broxton PD, Zeng X, Scheftic W, Troch PA. A MODISbased $1 \mathrm{~km}$ maximum green vegetation fraction dataset. J Appl Meteorol Clim. 2014;53:1996-2004. https://doi. org/10.1175/JAMC-D-13-0356.1

22. Hijmans RJ, Cameron SE, Parra JL, Jones PG, Jarvis A. Very high resolution interpolated climate surfaces for global land areas. J Appl Meteorol. 2005;25:1965-78. https://doi. org/10.1002/joc. 1276

23. Basille M, Calenge C, Marboutin E, Andersen R, Gaillard JM. Assessing habitat selection using multivariate statistics: Some refinements of the ecological-niche factor analysis. Ecol Modell. 2008;211:233-40. https://doi.org/10.1016/j. ecolmodel.2007.09.006

24. Calenge $\mathbf{C}$. The package "adehabitat" for the R software: A tool for the analysis of space and habitat use by animals. Ecol Modell. 2006;197:516-9. https://doi.org/10.1016/j. ecolmodel.2006.03.017

25. Hirzel AH, Hausser J, Chessel D, Perrin N. Ecologicalniche factor analysis: How to compute habitat-suitability maps without absence data? Ecology. 2002;83:2027-36. https://doi.org/10.1890/0012-9658(2002)083[2027:ENFAH T]2.0.CO;2

26. Phillips SJ, Anderson RP, Schapire RE. Maximum entropy modeling of species geographic distributions. Ecol Model. 2006;190:231-59. https://doi.org/10.1016/j.ecolmodel.2005. 03.026

27. Stockwell D. The GARP modelling system: Problems and solutions to automated spatial prediction. Int J Geogr Inf Sci. 1999;13:143-58. https://doi.org/10.1016/j.ecolmodel.2005. 03.026
28. Merow C, Smith MJ, Silander JA. A practical guide to MaxEnt for modeling species' distributions: What it does, and why inputs and settings matter. Ecography. 2013;36:105869. https://doi.org/10.1111/j.1600-0587.2013.07872.x

29. Peterson AT, Papeş M, Soberón J. Rethinking receiver operating characteristic analysis applications in ecological niche modeling. Ecol Model. 2008;213:63-72. https://doi. org/10.1016/j.ecolmodel.2007.11.008

30. Labruna MB, Mattar S, Nava S, Venzal JM, Dolz G, Abarca K, et al. Rickettsioses in Latin America, Caribbean, Spain and Portugal. Revista MVZ Córdoba. 2011;16:243557.

31. Mastropaolo M, Nava S, Guglielmone AA, Mangold AJ. Biological differences between two allopatric populations of Amblyomma cajennense (Acari: Ixodidae) in Argentina. Exp Appl Acarol. 2011;53:769-74. https://doi.org/10.1007/ s10493-010-9404-6

32. Estrada-Peña A, Tarragona EL, Vesco U, Meneghi D de, Mastropaolo M, Mangold AJ, et al. Divergent environmental preferences and areas of sympatry of tick species in the Amblyomma cajennense complex (Ixodidae). Int J Parasitol. 2014;44:1081-9. https://doi.org/10.1016/j. ijpara.2014.08.007

33. Rivera-Páez FA, Labruna MB, Martins TF, Pérez JE, Castaño-Villa GJ, Ossa-López PA, et al. Contributions to the knowledge of hard ticks (Acari: Ixodidae) in Colombia. Ticks Tick Borne Dis. 2018;9:57-66. http://doi.org/10.1016/j. ttbdis.2017.10.008

34. Álvarez C, Bonilla M, Chacón G. Distribución de la garrapata Amblyomma cajennense (Acari: Ixodidae) sobre Bos taurus y Bos indicus en Costa Rica. Revista de Biología Tropical. 2000;48:129-35.

35. Martins TF, Barbieri AR, Costa FB, Terassini FA, Camargo LM, Peterka CR, et al. Geographical distribution of Amblyomma cajennense (sensu lato) ticks (Parasitiformes: Ixodidae) in Brazil, with description of the nymph of $A$. cajennense (sensu stricto). Parasit Vectors. 2016;9:186. https://doi.org/10.1186/s13071-016-1460-2

36. Strey OF, Teel PD, Longnecker MT, Needham GR. Survival and water-balance characteristics of unfed adult Amblyomma cajennense (Acari: Ixodidae). J Med Entomol. 1996;33:63-73.

37. Szabó MP, Pinter A, Labruna MB. Ecology, biology and distribution of spotted-fever tick vectors in Brazil. Front Cell Infect Microbiol. 2013;3:27. https://doi.org/10.3389/ fcimb.2013.00027 
Cuadro suplementario 1. Registros únicos de la presencia del complejo Amblyomma cajennense en Colombia

\begin{tabular}{|c|c|c|c|}
\hline Punto & Departamento & Latitud & Longitud \\
\hline 1 & Antioquia & 8,1239 & $-76,5811$ \\
\hline 2 & Antioquia & 8,1309 & $-76,5522$ \\
\hline 3 & Antioquia & 8,1365 & $-76,5497$ \\
\hline 4 & Antioquia & 7,900 & $-76,65$ \\
\hline 5 & Antioquia & 8,129 & $-76,5734$ \\
\hline 6 & Antioquia & 6,491 & $-74,8426$ \\
\hline 7 & Antioquia & 8,1486 & $-76,5319$ \\
\hline 8 & Antioquia & 8,5361 & $-76,5742$ \\
\hline 9 & Antioquia & 8,5415 & $-76,5727$ \\
\hline 10 & Antioquia & 8,5482 & $-76,5738$ \\
\hline 11 & Antioquia & 8,5487 & $-76,5696$ \\
\hline 12 & Antioquia & 8,5166 & $-76,6666$ \\
\hline 13 & Antioquia & 8,75 & $-76,517$ \\
\hline 14 & Antioquia & 6,4847 & $-75,02$ \\
\hline 15 & Antioquia & 8,1104 & $-76,5357$ \\
\hline 16 & Arauca & 7,0136 & $-71,7774$ \\
\hline 17 & Caldas & 5,45 & $-74,7$ \\
\hline 18 & Casanare & 5,1833 & $-72,5393$ \\
\hline 19 & Cesar & 10,4171 & $-73,584$ \\
\hline 20 & Córdoba & 8,8505 & $-76,4263$ \\
\hline 21 & Córdoba & 8,8949 & $-76,3537$ \\
\hline 22 & Córdoba & 8,8211 & $-75,5008$ \\
\hline 23 & Córdoba & 9,2333 & $-75,8166$ \\
\hline 24 & Córdoba & 8,8366 & $-76,3375$ \\
\hline 25 & Córdoba & 8,758 & $-75,8833$ \\
\hline 26 & Córdoba & 8,5008 & $-75,5072$ \\
\hline 27 & Córdoba & 8,6276 & $-75,8829$ \\
\hline 28 & Cundinamarca & 5,043 & $-74,4449$ \\
\hline 29 & Cundinamarca & 4,5203 & $-74,5394$ \\
\hline 30 & Cundinamarca & 4,9833 & $-74,4833$ \\
\hline 31 & Cundinamarca & 5,046 & $-74,4635$ \\
\hline 32 & Cundinamarca & 4,9672 & $-74,5149$ \\
\hline 33 & Cundinamarca & 5,05 & $-74,5$ \\
\hline 34 & Cundinamarca & 5,0391 & $-74,5026$ \\
\hline 35 & Cundinamarca & 4,3166 & $-74,7833$ \\
\hline 36 & Cundinamarca & 5,017 & $-74,467$ \\
\hline 37 & Cundinamarca & 4,5835 & $-74,5999$ \\
\hline 38 & Cundinamarca & 4,9834 & $-74,4999$ \\
\hline 39 & Cundinamarca & 5,0415 & $-74,47$ \\
\hline 40 & Cundinamarca & 5,0547 & $-74,5333$ \\
\hline 41 & Cundinamarca & 4,4666 & $-74,6333$ \\
\hline 42 & Cundinamarca & 5,015 & $-74,474$ \\
\hline 43 & Huila & 3,2222 & $-75,2369$ \\
\hline
\end{tabular}




\begin{tabular}{|c|c|c|c|}
\hline Punto & Departamento & Latitud & Longitud \\
\hline 44 & Huila & 2,5833 & $-75,3$ \\
\hline 45 & Huila & 3,1538 & $-75,0538$ \\
\hline 46 & Huila & 2,6833 & $-75,3333$ \\
\hline 47 & Huila & 3,3666 & $-74,8$ \\
\hline 48 & Huila & 2,3833 & $-75,9$ \\
\hline 49 & Huila & 2,45 & $-75,7666$ \\
\hline 50 & Huila & 2,7833 & $-75,25$ \\
\hline 51 & Huila & 3,0675 & $-75,1386$ \\
\hline 52 & Huila & 2,4833 & $-75,7333$ \\
\hline 53 & Huila & 3,22 & $-75,2191$ \\
\hline 54 & Huila & 2,6666 & $-75,5166$ \\
\hline 55 & Magdalena & 11,2383 & $-74,1361$ \\
\hline 56 & Meta & 4,5667 & $-71,3333$ \\
\hline 57 & Meta & 4,0833 & $-72,95$ \\
\hline 58 & Meta & 3,3818 & $-73,8738$ \\
\hline 59 & Meta & 4,0724 & $-73,5815$ \\
\hline 60 & Meta & 4,2992 & $-72,3783$ \\
\hline 61 & Meta & 4,1426 & $-73,6294$ \\
\hline 62 & Sucre & 9,6666 & $-75,5$ \\
\hline 63 & Sucre & 9,7333 & $-75,5333$ \\
\hline 64 & Sucre & 9,726 & $-75,6484$ \\
\hline 65 & Tolima & 3,5833 & $-75,3833$ \\
\hline 66 & Tolima & 3,7166 & $-75,4833$ \\
\hline 67 & Tolima & 3,7833 & $-75,2$ \\
\hline 68 & Tolima & 3,5416 & $-74,8991$ \\
\hline 69 & Tolima & 5,1916 & $-74,7426$ \\
\hline 70 & Tolima & 4,2 & $-75,1166$ \\
\hline 71 & Tolima & 3,9333 & $-75,2166$ \\
\hline 72 & Tolima & 4,2 & $-74,65$ \\
\hline 73 & Tolima & 4,7166 & $-74,9166$ \\
\hline 74 & Valle del Cauca & 3,5394 & $-76,3036$ \\
\hline
\end{tabular}

